Pacific Journal of Mathematic 


\title{
FINITE SIGNED MEASURES ON FUNCTION SPACES
}

\section{A. J. VAN HAAGEN}

\begin{abstract}
Some results for probability measures on function spaces are extended to finite signed measures (FSM's). In particular FSM's on the space of continuous functions and right-continuous functions with left-hand limits are patched together by a procedure of Stroock and Varadhan. Given an increasing sequence of stopping times the procedure is carried out repeatedly. A sequence of transition functions and, an extension result for the linear maps associated with these transition functions are obtained.
\end{abstract}

Introduction. In recent years some papers have appeared related to signed measures on function spaces (see [3] and [4]). This paper extends certain results for probability measures on function spaces to finite signed measures (FSM's) on such spaces. A more detailed discussion can be found in [8].

In part I we introduce conditional FSM's and consider the existence of a regular conditional distribution (RCD) of an FSM on a standard measurable space mimicking Chapter V of [5]. Further, the Jordan decomposition of an RCD is investigated. We then consider a sequence of transition functions and associate linear maps between Banach spaces of FSM's with these transition functions. An extension result for the linear maps is obtained.

In part II FSM's on $\Omega=C([0, \infty) ; S)$ and $\Omega=D([0, \infty) ; S)$ with $S$ a separable metric space are patched together by the procedure used in [6] for probability measures on $C\left([0, \infty) ; R^{d}\right)$. In fact, if $\mathscr{C}^{s}$ is the $\sigma$-field on $\Omega$ generated by the coordinate projections $\left\{X_{t}, t \geqq s\right\}$ and $\tau$ an $s$-stopping time with respect to the $\sigma$-fields $\mathscr{A}_{t}^{s}=\sigma\left\{X_{u}, s \leqq u \leqq t\right\}$, then an FSM on $\mathscr{L}_{\tau}^{s}$ is patched together with a family $\left\{\mu_{\omega}\right\}_{\omega \in \Omega}$ of FSM's where $\mu_{\omega}$ has domain $\mathscr{M}^{\tau(\omega)}$ if $\tau(\omega)<\infty$.

If $S$ is a complete separable metric space, $\left(\Omega, \mathscr{C}^{s}\right)$ and $\left(\Omega, \mathscr{L}_{\tau}^{s}\right)$ are standard measurable spaces. In this case any FSM on $\left(\Omega, \mathscr{C}^{s}\right)$ with an RCD given $\mathscr{C}_{\tau}^{8}$ can be thought of as obtained by patching. If $\tau_{0}, \tau_{1}, \cdots$ is an increasing sequence of $s$-stopping times $\mathscr{L}_{\tau_{0}}^{s}$, $\mathscr{C}_{\tau_{1}}^{s}, \cdots$ is the corresponding sequence of $\sigma$-fields and, given families of FSM's $\left\{\mu_{n \omega}\right\}_{\omega}$ on $\mathscr{\mathscr { C }}^{\tau_{n}(\omega)}$ for each $n$ with the right properties the patching procedure can be applied repeatedly. We have in fact an associated sequence of transition functions and the results of part I apply.

Basic facts on FSM's are taken from [2]. 
Part I. RCD's of FSM's on Standard Measurable Spaces

1. Conditional FSM's, Let $(\Omega, \mathscr{F}, \mu)$ be an FSM space and $\Sigma$ a sub $\sigma$-field of $\mathscr{F}$. If $\mu_{\Sigma}$ is the restriction of $\mu$ to $\Sigma$, then a $\mu_{\Sigma^{-}}$ null set is not necessarily a $\mu$-null set. However we can prove the following.

THEOREM 1.1. If each $\mu_{\Sigma}$-null set of $\Omega$ is also a $\mu$-null set, and if $B$ is any $\mathscr{F}$-measurable set, then there exists a $\Sigma$-measurable real function $\mu(B \mid \Sigma)$ such that for all $A \in \Sigma$

$$
\mu(A \cap B)=\int_{A} \mu(B \mid \Sigma) d \mu_{\Sigma} .
$$

Any two such functions for given $B$ must coincide $\mu$-a.e. $\mu(B \mid \Sigma)$ is called the conditional FSM of $B$ given $\Sigma$.

Proof. For $B \in \mathscr{F}$ we define an FSM $\lambda$ on $\Sigma$ by $\lambda(A)=\mu(A \cap B)$ for all $A \in \Sigma$. As $\lambda$ is absolutely continuous with respect to $\mu_{\Sigma}$ it follows by the Radon-Nikodym theorem that there exists a $\Sigma$ measurable real function $f$ on $\Omega$ such that

$$
\mu(A \cap B)=\int_{A} f d \mu_{\Sigma} \quad \text { for all } A .
$$

We can take $f$ to be $\mu(B \mid \Sigma)$.

THEOREM 1.2. With the assumptions on $\mu$ of Theorem 1.1 let $\left\{B_{n}\right\}_{1}^{\infty}$ be a sequence of disjoint $\mathscr{F}$-measurable sets. Then

$$
\mu\left(\bigcup_{n} B_{n} \mid \Sigma\right)=\sum_{n} \mu\left(B_{n} \mid \Sigma\right) \quad \mu \text {-a.e. }
$$

Proof. Let $\mu=\mu^{+}-\mu^{-}$be the Jordan decomposition of $\mu$ and let $\left|\mu_{\Sigma}\right|$ be the total variation of $\mu_{\Sigma}$. If $B \in \mathscr{F}$, there exist $\Sigma$ measurable real functions $h_{1}(B \mid \Sigma) \geqq 0$ and $h_{2}(B \mid \Sigma) \geqq 0$ such that for all $A \in \Sigma$

$$
\begin{aligned}
& \mu^{+}(A \cap B)=\int_{A} h_{1}(B \mid \Sigma) d\left|\mu_{\Sigma}\right|, \\
& \mu^{-}(A \cap B)=\int_{A} h_{2}(B \mid \Sigma) d\left|\mu_{\Sigma}\right| .
\end{aligned}
$$

If we put $h(B \mid \Sigma)=h_{1}(B \mid \Sigma)-h_{2}(B \mid \Sigma)$, then $|\mu(B \mid \Sigma)|=|h(B \mid \Sigma)| \mu-$ a.e. It now follows easily that for all $A \in \Sigma$

$$
\int_{\Delta} \sum_{n} \mu\left(B_{n} \mid \Sigma\right) d \mu_{\Sigma}=\int_{A} \mu\left(\bigcup_{n} B_{n} \mid \Sigma\right) d \mu_{\Sigma}
$$


REMARK 1.3. Note that for all $B \in \mathscr{F},|\mu(B \mid \Sigma)| \leqq h_{1}(\Omega \mid \Sigma)+$ $h_{2}(\Omega \mid \Sigma)$ except on a $\mu$-null set $N$ (which depends on $B$ in general).

Definition 1.4. Let $(\Omega, \mathscr{F}, \mu)$ be an FSM space, $\Sigma$ a sub $\sigma$-field of $\mathscr{F}$ and $R$ the set of real numbers. The function $Q: \Omega \times \mathscr{F} \rightarrow R$ is called an RCD of $\mu$ given $\Sigma$ if it has the properties:

(i) For each $B \in \mathscr{F}, \omega \rightarrow Q(\omega, B)$ is a $\Sigma$-measurable map from $\Omega$ into $R$.

(ii) For each $\omega \in \Omega, Q(\omega, \cdot)$ is an FSM on $\mathscr{F}$ with $Q(\omega, \Omega)=1$.

(iii) For all $A \in \Sigma$ and $B \in \mathscr{F}$

$$
\mu(A \cap B)=\int_{A} Q(\omega, B) d \mu_{\Sigma}(\omega) .
$$

In $\S 3$ we give conditions under which $Q$ exists.

LEMMA 1.5. Let $(\Omega, \mathscr{F}, \mu)$ be a countably generated FSM space and $\Sigma$ a sub $\sigma$-field of $\mathscr{F}$. If $Q_{1}$ and $Q_{2}$ are RCD's of $\mu$ given $\Sigma$, then $Q_{1}(\omega, B)=Q_{2}(\omega, B)$ for all $B \in \mathscr{F}$ except for $\omega$ in a $\mu-n u l l$ set of $\Sigma$ which is independent of $B$.

Proof. The proof is the same as for regular conditional probability distributions (RCPD's).

2. Extension theorems for FSM's. In this section we generalize theorems for probability measures appearing in Chapter $\mathrm{V}$ of [5] to theorems on FSM's.

DEFINITION 2.1. Let $\left\{\mathscr{B}_{n}\right\}_{1}^{\infty}$ be an increasing family of $\sigma$-fields on the space $X$. The family of FSM's $\left\{\mu_{n}\right\}_{1}^{\infty}$ is said to be consistent if $\mu_{n}$ is defined on $\mathscr{B}_{n}$ and for all $A \in \mathscr{B}_{n}$ and $m \geqq n, \mu_{n}(A)=\mu_{m}(A)$. The family is said to be uniformly bounded if $\sup _{n}\left|\mu_{n}\right|(X)<\infty$. proof.

We will need the following simple lemma of which we omit the

LEMMA 2.2. If $\left\{\mu_{n}\right\}_{1}^{\infty}$ is a uniformly bounded consistent family of FSM's on the $\sigma$-fields $\left\{\mathscr{B}_{n}\right\}_{1}^{\infty}$, then there exists a unique finitely additive set function $\mu$ on the field $\mathrm{U}_{n} \mathscr{B}_{n}$ with the properties:

(i) $\mu(A)=\mu_{n}(A)$ for all $A \in \mathscr{B}_{n}$ and $n=1,2, \cdots$.

(ii) Given $\varepsilon>0$, there exists a positive integer $n_{0}$ such that $|\mu|(A)-\left|\mu_{n}\right|(A)<\varepsilon$ for all $A \in \mathscr{B}_{n}$ if $n \geqq n_{0}$.

TheOREM 2.3 (analogue of Theorem 4.1 on p. 141 of [5]). Let $(X, \mathscr{B})$ be a measurable space and for each $n=1,2, \cdots \mathscr{B}_{n}$ is a 
sub $\sigma$-field of $\mathscr{B}$ such that

(i) $\mathscr{B}_{1} \subseteq \mathscr{B}_{2} \subseteq \cdots$, and $\cup_{n} \mathscr{B}_{n}$ generates $\mathscr{B}$.

(ii) $\left(X, \mathscr{B}_{n}\right)$ is a standard measurable space for $n=1,2, \cdots$.

Then, in order that every uniformly bounded consistent sequence of FSM's on $\mathscr{B}_{1}, \mathscr{B}_{2}, \cdots$ be extendable to an FSM on $\mathscr{B}$, it is necessary and sufficient that $\bigcap_{1}^{\infty} A_{n} \neq \varnothing$ for each decreasing sequence $\left\{A_{n}\right\}_{1}^{\infty}$ of subsets of $X$ such that $A_{n}$ is an atom of $\mathscr{B}_{n}$ for all $n$. If this is the case, $(X, \mathscr{B})$ is also standard, and if $\left\{\mu_{n}\right\}_{1}^{\infty}$ is such a sequence of FSM's, the extension is unique. Moreover, $|\mu|(X)=$ $\sup _{n}\left|\mu_{n}\right|(X)$.

Proof. The necessity is proved in [5]. In order to prove the sufficiency we first prove the analogue for FSM's of Theorem 3.1 on p. 138 of [5]. We use the notation of [5] and make the further assumption that $\sup _{n}\left|\mu_{n}\right|\left(Z_{n}\right)<\infty$.

Let $\mu$ be the finitely additive bounded set function on $\mathscr{F}$ analogous in the obvious way to $\mu$ on p. 139 of [5]. We will show that if $\left\{A_{n}\right\}_{1}^{\infty}$ is a decreasing sequence in $\mathscr{F}$ and if $|\mu|\left(A_{n}\right) \geqq \delta>0$ for all $n$ and some $\delta>0$, then $\bigcap_{n} A_{n} \neq \varnothing$. Without loss of generality we may assume $A_{n} \in \tilde{\mathscr{D}}_{n}$ for $n=1,2, \cdots$. For $n=1,2, \cdots$ and $\mathfrak{l}=0,1, \cdots$ we can write $A_{n}=\widetilde{\varphi}_{n+\mathfrak{l}}^{-1}\left(B_{n, \mathfrak{l}}\right)$ where $B_{n, \mathfrak{l}} \in \mathscr{D}_{n+\mathfrak{r}}$. For each $n$ and $\mathfrak{l}$ there exists a compact set $K_{n, \mathfrak{l}}^{0} \subseteq B_{n, \mathfrak{r}}$ in $\mathscr{D}_{n+\mathfrak{r}}$ such that $\left|\mu_{n+1}\right|\left(B_{n, 1}-K_{n, 1}^{0}\right) \leqq \delta / 4^{n}$. Put $\widetilde{K}_{n, \mathrm{l}}=\widetilde{\varphi}_{n+1}^{-1}\left(K_{n, 1}^{0}\right)$. By Theorem 2.6 on p. 136 of [5], $Z$ is compact and $\widetilde{\varphi}_{n+1}$ is continuous. Hence, $\widetilde{K}_{n, 1}$ is compact. Moreover, $\widetilde{K}_{n, \mathfrak{l}} \subseteq A_{n}$ for $\mathfrak{l}=0,1, \cdots$ and

(*) $\quad\left|\mu_{\mathscr{D}_{n+1}}\right|\left(A_{n}-\widetilde{K}_{n, 1}\right) \leqq \delta / 4^{n} \quad$ for all $n$ and $\mathfrak{l}$.

Now $\left\{\mu_{\mathscr{T}_{j}}\right\}_{1}^{\infty}$ is a uniformly bounded consistent family of FSM's. By Lemma 2.2 there exists for each $n$ an $\mathfrak{I}(n)>0$ such that $|\mu|\left(A_{n}\right.$ $\left.\widetilde{K}_{n, \mathrm{I}(n)}\right)-\left|\mu_{\tilde{\mathscr{C}}_{n+\mathrm{I}(n)}}\right|\left(A_{n}-\widetilde{K}_{n, \mathrm{I}(n)}\right)<\delta / 4^{n+1}$. Together with $(*)$ we obtain $|\mu|\left(A_{n}-\widetilde{K}_{n, r(n)}\right)<5 \delta / 4^{n+1}$.

Put $K_{n}=\bigcap_{j=1}^{n} \widetilde{K}_{j, r(j)}$ for $n=1,2, \cdots$. As in [5] it follows that $\bigcap_{n} A_{n} \neq \varnothing$. Thus $\mu$ is countably additive on $\mathscr{F}$. Therefore $\mu$ has a unique extension to an FSM on $\mathscr{D}$, which we also denote by $\mu$, and $|\mu|(Z)=\sup _{n}\left|\mu_{n}\right|\left(Z_{n}\right)$.

We can now prove the analogue for FSM's of Theorem 3.2 on p. 139 of [5]. This proof and the proof of the theorem at hand go through in the same way as in [5]. For details see [8].

The next theorem is Kolmogorov's extension theorem for FSM's. Its proof is similar to Parthasarathy's proof for probability measures. With the notation of Theorem 5.1 on p. 144 of [5] we have the following. 
THEOREM 2.4. Let $\left(X_{\alpha}, \mathscr{B}_{\alpha}\right), \alpha \in I$, be standard separable measurable spaces. If $\left\{\mu_{F}: F \cong I, F\right.$ finite $\}$ is a uniformly bounded consistent family of FSM's, then there exists a unique FSM $\mu$ on $\mathscr{B}^{I}$ such that $\mu_{F}(A)=\mu\left(\pi_{I F}^{-1}(A)\right)$ for all $A \in \mathscr{B}^{F}$ and all finite $F \cong I$. Moreover, $|\mu|\left(X^{I}\right)=\sup _{F \subseteq_{I} \text { finite }}\left|\mu_{F}\right|\left(X^{F^{\prime}}\right)$.

3. The existence of an RCD of an FSM.

TheOREm 3.1 (analogue of Theorem 7.1 on p. 145 of [5]). Let $(X, \mathscr{B})$ be a standard measurable space and $\Sigma$ a sub $d$-field of $\mathscr{B}$. Let $\mu$ be an FSM on $\mathscr{B}$. The condition that a $\mu_{\Sigma}-n u l l$ set of $X$ is a $\mu-n u l l$ set is necessary and sufficient for the existence of an RCD of $\mu$ given $\Sigma$.

Proof. The necessity follows immediately from the definition. If $X$ is countable the sufficiency is a direct consequence of Theorems 1.1 and 1.2. Let $X$ be uncountable. As is shown in [5] there exists an increasing sequence of finite $\sigma$-fields $\left\{\mathscr{B}_{n}\right\}_{1}^{\infty}$ such that $\bigcup_{n} \mathscr{B}_{n}$ generates $\mathscr{B}$ and any uniformly bounded consistent sequence of FSM's on $\left\{\mathscr{B}_{n}\right\}_{1}^{\infty}$ is extendable to an FSM on $\mathscr{B}$.

Following [5] it is easy to see that for $n=1,2, \cdots$ there exists a function $Q_{n}: X \times \mathscr{B}_{n} \rightarrow R$ such that

(i) for each $A \in \mathscr{B}_{n}, x \rightarrow Q_{n}(x, A)$ is $\Sigma$-measurable.

(ii) for each $x \in X, Q_{n}(x, \cdot)$ is an FSM on $\mathscr{B}_{n}$ and $Q_{n}(x, X)=1$.

(iii) for all $A \in \Sigma$ and $B \in \mathscr{B}_{n}$

$$
\mu(A \cap B)=\int_{A} Q_{n}(x, B) d \mu_{\Sigma}(x) .
$$

By the argument in [5] and Remark 1.3 there exists a $\mu$-null set $N \in \Sigma$ such that for each $x \in X-N, Q_{1}(x, \cdot), Q_{2}(x, \cdot), \cdots$ is a uniformly bounded consistent sequence of FSM's on $\mathscr{B}_{1}, \mathscr{B}_{2}, \ldots$. Thus for each $x \in X-N$ there exists a unique FSM, $Q_{x}$, on $\mathscr{B}$ such that $Q_{x}(A)=Q_{n}(x, A)$ for all $A \in \mathscr{B}_{n}$ and $n=1,2, \cdots$. Define the function $Q$ on $X \times \mathscr{B}$ by

$$
Q(x, B)= \begin{cases}Q_{x}(B) & \text { if } B \in \mathscr{B} \text { and } x \in X-N, \\ P(B) & \text { if } B \in \mathscr{B} \text { and } x \in N,\end{cases}
$$

where $P$ is any fixed probability measure on $\mathscr{B}$. Now $|Q|(x, X)=$ $\sup _{n}\left|Q_{n}\right|(x, X)$ if $x \in X-N$ and, consequently, Remark 1.3 implies that $x \rightarrow|Q|(x, X)$ is $\mu_{\Sigma}$-integrable. That $Q$ is an $\mathrm{RCD}$ of $\mu$ given $\Sigma$ follows now as in [5].

CoRollary 3.2. The map $x \rightarrow|Q|(x, B)$, from $X$ into $R$, is $\mu_{\Sigma^{-}}$ integrable for all $B \in \mathscr{B}$. 
Proof. Let $\mathscr{F}$ be a countable field generating $\mathscr{B}$. Then $|Q|(x, B)=\sup \sum_{i=1}^{n}\left|Q\left(x, B_{i}\right)\right|$, where the sup is taken over all finite sequences $\left\{B_{i}\right\}$ of disjoint sets in $\mathscr{F}$ such that $B_{i} \cong B$. The assertion now follows.

COROLLARY 3.3. If $\Sigma$ is countably generated, then there exists a e-null set $N \in \Sigma$ such that for all $A \in \Sigma$ and $B \in \mathscr{B}, Q(x, A B)=$ $I_{A}(x) Q(x, B)$ if $x \in X-N$.

Proof. Clearly, if $A \in \Sigma$ and $B \in \mathscr{B}, Q(x, A B)=I_{A}(x) Q(x, B)$ except for $x$ in a $\mu$-null set in $\Sigma$ depending on $A$ and $B$. Now both $\Sigma$ and $\mathscr{B}$ are countably generated and the proof is completed by a standard argument.

The next theorem concerns the Jordan decomposition of an RCD.

THEOREM 3.4. Let $(X, \mathscr{B})$ be a standard measurable space and $\Sigma$ a countably generated sub $\sigma$-field of $\mathscr{B}$.

Let $\mu$ be an FSM on $\mathscr{B}$ with an RCD, $Q$, given $\Sigma$. If $Q(x, \cdot)=$ $Q^{+}(x, \cdot)-Q^{-}(x, \cdot)$ is the Jordan decomposition of $Q(x, \cdot)$, then there exists probability measures $P_{1}$ and $P_{2}$ on $\mathscr{B}$ with RCPD's $Q_{1}$ and $Q_{2}$ given $\Sigma$, respectively, and there exists a $\mu$-null set $N \in \Sigma$ such that for all $B \in \mathscr{B}$

$$
Q(x, B)=Q^{+}(x, X) Q_{1}(x, B)-Q^{-}(x, X) Q_{2}(x, B)
$$

if $x \in X-N$.

Proof. From $Q^{+}(x, B)=1 / 2\{|Q|(x, B)+Q(x, B)\}$ and $Q^{-}(x, B)=$ $1 / 2\{|Q|(x, B)-Q(x, B)\}$ it follows that the maps $x \rightarrow Q^{+}(x, B)$ and $x \rightarrow Q^{-}(x, B)$ are $\mu_{\Sigma^{-}}$integrable for each $B \in \mathscr{B}$. There exists a $\mu$-null set $N_{1} \in \Sigma$ such that $Q(x, A B)=I_{A}(x) Q(x, B)$ for all $A \in \Sigma$ and $B \in \mathscr{B}$ if $x \in X-N_{1}$. Hence, $Q^{+}(x, A B)=I_{A}(x) Q^{+}(x, B)$ and $Q^{-}(x, A B)=$ $I_{A}(x) Q^{-}(x, B)$ if $x \in X-N_{1}$.

Let $P$ be any probability measure on $(X, \mathscr{B})$ such that $\mu$ and $P$ are absolutely continuous with respect to each other and let $Q^{\prime}$ be an RCPD of $P$ given $\Sigma$. We set $F_{1}=\left\{x: Q^{+}(x, X)=0\right\}$ and $F_{2}=$ $\left\{x: Q^{-}(x, X)=0\right\}$ and define, for all $B \in \mathscr{B}$,

$$
Q_{1}(x, B)= \begin{cases}\frac{Q^{+}(x, B)}{Q^{+}(x, X)} & \text { if } \quad x \in X-F_{1}, \\ Q^{\prime}(x, B) & \text { if } \quad x \in F_{1},\end{cases}
$$




$$
Q_{2}(x, B)=\left\{\begin{array}{lll}
\frac{Q^{-}(x, B)}{Q^{-}(x, X)} & \text { if } \quad x \in X-F_{2}, \\
Q^{\prime}(x, B) & \text { if } \quad x \in F_{2} .
\end{array}\right.
$$

Let probability measures $P_{1}$ and $P_{2}$ on $\mathscr{B}$ be defined by $P_{1}(B)=$ $\int Q_{1}(x, B) d P(x)$ and $P_{2}(B)=\int Q_{2}(x, B) d P(x)$. Clearly, $Q_{1}$ and $Q_{2}$ are RCPD's given $\Sigma$ of $P_{1}$ and $P_{2}$ respectively. Moreover, for all $A \in \Sigma$ and $B \in \mathscr{B}$,

$$
\mu(A \cap B)=\int_{A}\left[Q^{+}(x, X) Q_{1}(x, B)-Q^{-}(x, X) Q_{2}(x, B)\right] d \mu_{\Sigma}(x) .
$$

The proof is complete.

\section{Sequences of transition functions.}

DEFINITION 4.1. Let $\left\{\mathscr{B}_{n}\right\}_{0}^{\infty}$ be an increasing sequence of $\sigma$-fields on the space $X$. For all $n>m \geqq 0$ let the functions $f_{m, n}: X \times \mathscr{B}_{n} \rightarrow R$ have the properties:

(i) For each $B \in \mathscr{B}_{n}, x \rightarrow f_{m, n}(x, B)$ is a $\mathscr{B}_{m}$-measurable map from $X$ into $R$.

(ii) For each $x \in X, f_{m, n}(x, \cdot)$ is an FSM on $\mathscr{B}_{n}$ and $f_{m, n}(x, X)=1$.

(iii) $\sup _{x \in X}\left|f_{m, n}\right|(x, X)<\infty$.

(iv) For all $x \in X, A \in \mathscr{\mathscr { B }}_{m}$ and $B \in \mathscr{\mathscr { B }}_{n}, f_{m, n}(x, A B)=I_{A}(x) f_{m, n}(x, B)$. $f_{m, n}$ will be called a transition function from $\left(X, \mathscr{B}_{m}\right)$ to $\left(X, \mathscr{B}_{n}\right)$. For $f_{n-1, n}$ we write $f_{n}$.

LEMMA 4.2. Let $\left\{f_{n}\right\}_{1}^{\infty}$ be a sequence of transition functions with respect to the $\sigma$-fields $\left\{\mathscr{B}_{n}\right\}_{0}^{\infty}$. For $0 \leqq n \leqq m$ define the functions $f_{m, n}$ on $X \times \mathscr{B}_{n}$ by $f_{m, n}(x, B)=I_{B}(x)$. Then the functions $f_{m, n}: X \times$ $\mathscr{B}_{n} \rightarrow R$ inductively defined for $n>m \geqq 0$ by

$$
f_{m, n}(x, B)=\int f_{n}\left(x^{\prime}, B\right) f_{m, n-1}\left(x, d x^{\prime}\right)
$$

are transition functions and if $\sup _{x}\left|f_{n}\right|(x, X)=\alpha_{n}$, then $\sup _{x}\left|f_{m, n}\right|(x, X) \leqq \alpha_{m+1} \cdots \alpha_{n}$. Moreover, for $B \in \mathscr{B}_{n}$ and $n>m \geqq 0$, $f_{m, n}(x, B)=f_{m, n+1}(x, B)$ and

$$
f_{m, n}(x, B)=\int f_{m+1, n}\left(x^{\prime}, B\right) f_{m+1}\left(x, d x^{\prime}\right) .
$$

Proof. This is straight forward and will be omitted. We only observe that for $n>m \geqq 0$ and $B \in \mathscr{B}_{n}$,

$$
f_{m, n}(x, B)=\int\left[\cdots\left[\int f_{n}\left(x_{n-1}, B\right) f_{n-1}\left(x_{n-2}, d x_{n-1}\right)\right] \cdots\right] f_{m+1}\left(x, d x_{m+1}\right) .
$$


Definition 4.3. For $n=0,1, \cdots$ let $M_{n}=M\left(X, \mathscr{B}_{n}\right)$ be the set of FSM's on $\left(X, \mathscr{B}_{n}\right)$. With the usual operations and the total variation norm $M_{n}$ is a Banach space. Let $\left\{f_{n}\right\}_{1}^{\infty}$ be transition functions as in Lemma 4.2. For $n=1,2 \cdots$ define the linear maps $L_{n}: M_{n-1} \rightarrow M_{n}$ by $L_{n}(\mu)(B)=\int f_{n}(x, B) d \mu(x) . \quad L_{n} \quad$ is injective and $\left\|L_{n}\right\| \leqq \alpha_{n}$. For $n>m \geqq 0$ let $L_{m, n}=L_{n} L_{n-1} \cdots L_{m+1}$. Then $L_{m, n}(\mu)(B)=\int f_{m, n}(x, B) d \mu(x)$ and $\left\|L_{m, n}\right\| \leqq \alpha_{m+1} \cdots \alpha_{n}$.

THEOREM 4.4. Let $(X, \mathscr{B})$ be a measurable space and $\left\{\mathscr{B}_{n}\right\}_{0}^{\infty}$ an increasing sequence of sub $\sigma$-fields of $\mathscr{B}$ as in Theorem 2.3 and satisfying the extension condition. Let $f_{1}, f_{2}, \cdots$ be a sequence of transition functions with respect to $\left\{\mathscr{B}_{n}\right\}_{0}^{\infty}$ such that $\alpha=\prod_{1}^{\infty} \sup _{x}\left|f_{n}\right|(x, X)<\infty$. Then for each $m=0,1, \cdots$ there exists a unique transition function $g_{m}$ from $\left(X, \mathscr{B}_{m}\right)$ to $(X, \mathscr{B})$ such that $g_{m}(x, B)=f_{m, n}(x, B)$ for all $x \in X, B \in \mathscr{B}_{n}$ and $n>m$. If $M$ is the Banach space of FSM's on $(X, \mathscr{B})$, then for each $m=0,1, \cdots$ there exists a unique injective linear map $T_{m}: M_{m} \rightarrow M$ with norm $\left\|T_{m}\right\| \leqq \alpha$ such that $T_{m}(\mu)(B)=$ $L_{m, n}(\mu)(B)$ for all $\mu \in M_{m}, B \in \mathscr{B}_{n}$ and $n>m$. Moreover, $g_{m}$ is an $\mathrm{RCD}$ of $T_{m}(\mu)$ given $\mathscr{B}_{m}$ for all $\mu \in M_{m}$.

Proof. Obviously, $f_{m, m+1}(x, \cdot), f_{m, m+2}(x, \cdot), \cdots$ is a uniformly bounded consistent sequence of FSM's on $\mathscr{B}_{m+1}, \mathscr{B}_{m+2}, \cdots$ for each $x$. There exists a unique FSM $g_{m}(x, \cdot)$ on $\mathscr{B}$ such that $g_{m}(x, B)=$ $f_{m, n}(x, B)$ for all $B \in \mathscr{B}_{n}$ and $n>m$. Moreover, $\sup _{x}\left|g_{m}\right|(x, X) \leqq \alpha$, and $g_{m}$ is a transition function from $\left(X, \mathscr{B}_{m}\right)$ to $(X, \mathscr{B})$.

For all $\mu \in M_{m}, B \in \mathscr{B}_{n}$ and $n>m \geqq 0$

$$
L_{m, n}(\mu)(B)=\int g_{m}(X, B) d \mu(X) .
$$

Thus $\left\{L_{m, n}(\mu)\right\}_{n=m+1}^{\infty}$ is a consistent sequence of FSM's such that $\sup _{n>m}\left|L_{m, n}(\mu)\right|(X) \leqq \alpha|\mu|(X)$. There exists a unique FSM on $\mathscr{B}$ which we will denote by $T_{m}(\mu)$ such that $T_{m}(\mu)(B)=L_{m, n}(\mu)(B)$ for all $B \in \mathscr{B}_{n}, n>m$. It is easily seen that $T_{m}: M_{m} \rightarrow M$ is a one-toone linear map with $\left\|T_{m}\right\| \leqq \alpha$. The last assertion follows from $\left(^{*}\right)$ and the above.

CoRollary 4.5. For $n=0,1, \cdots$ let $\mu_{n}$ denote the restriction to $\mathscr{B}_{n}$ of an element $\mu$ of $M$, then the map $P_{n}: M \rightarrow M$ defined by $P_{n}(\mu)=T_{n}\left(\mu_{n}\right)$ is a continuous linear projection with null space $\left\{\mu \in M: \mu_{n}=0\right\}$. Moreover, $P_{n}(\mu)(B)=\int g_{n}(x, B) d \mu(x)$ for all $\mu \in M$ and $B \in \mathscr{B}$, and $P_{n} P_{m}=P_{n}$ for all $m \geqq n$.

Part II. FSM's on the function spaces $C([0, \infty) ; S)$ and $D([0, \infty) ; S)$ 
In $\S \S 5$ and 6 the patching Theorem on p. 367 of [6] is generalized.

5. Extension of a $\tau$-basic family of FSM's.

Notations and Definitions 5.1, Let $S$ be a metric space. $C([0, \infty) ; S)$ will denote the space of all continuous functions on $[0, \infty)$ with values in $S . \quad D([0, \infty) ; S)$ denotes the space of all $S$ valued functions on $[0, \infty)$ that are right-continuous and have lefthand limits. The symbol $\Omega$ will be used both for $C([0, \infty) ; S)$ and $D([0, \infty) ; S)$. For each $t \in[0, \infty)$ we define the coordinate projection $X_{t}: \Omega \rightarrow S$ by $X_{t}(\omega)=\omega(t)$ for all $\omega \in \Omega$. Instead of $X_{t}$ we also write $X(t)$. The Borel $\sigma$-field of $S$ will be denoted by $\mathscr{B}(S)$ or $\mathscr{S}$. If $0 \leqq a \leqq t \leqq b<\infty$, then $\mathscr{A}_{b}^{a}=\sigma\left(X_{t}, a \leqq t \leqq b\right)$ is the $\sigma$-field on $\Omega$ generated by the maps $X_{t}, a \leqq t \leqq b$. If $b=\infty$ we write $\mathscr{M}^{a}$ for $\sigma\left(X_{t}, t \geqq a\right) . \quad \mathscr{C}_{b}^{a}\left(\mathscr{C}^{a}\right)$ is the semifield of subsets of $\Omega$ of the form $\left\{X_{t_{1}} \in \Gamma_{1}, \cdots, X_{t_{n}} \in \Gamma_{n}\right\}$, where $t_{1}, \cdots, t_{n}$ are points in $[a, b]$ $([a, \infty)), \Gamma_{1}, \cdots, \Gamma_{n}$ Borel sets in $S$ and $n$ any positive integer. Clearly, $\mathscr{M}_{b}^{a}=\sigma\left(\mathscr{C}_{b}^{a}\right)$ and $\mathscr{C}^{a}=\sigma\left(\mathscr{C}^{a}\right) . \quad \mathscr{C}^{\infty}$ and $\mathscr{M}^{\infty}$ will stand for $\{\varnothing, \Omega\}$.

If $s \geqq 0$, then an $s$-Markov time or $s$-stopping time is a map $\tau: \Omega \rightarrow[s, \infty]$ such that $\{\tau \leqq t\} \in \mathscr{M}_{t}^{s}$ for all $t \geqq s . \quad \mathscr{C}_{\tau}^{s}=\left\{A \in \mathscr{M}^{s}\right.$ : $A \cap\{\tau \leqq t\} \in \mathscr{A}_{t}^{s}$ for all $\left.t \geqq s\right\}$ is the $\sigma$-field of sets in $\mathscr{M}^{s}$ prior to $\tau$. If $\omega \in \Omega$, the atom of $\mathscr{L}_{\tau}^{s}$ containing $\omega$ is given by

$$
A_{\omega}= \begin{cases}\left\{\omega^{\prime} \in \Omega: X\left(t, \omega^{\prime}\right)=X(t, \omega), s \leqq t \leqq \tau(\omega)\right\} & \text { if } \tau(\omega)<\infty, \\ \left\{\omega^{\prime} \in \Omega: X(t, \omega)=X(t, \omega), t \geqq s\right\} & \text { if } \tau(\omega)=\infty .\end{cases}
$$

DEFINITION 5.2. A family of FSM's $\left\{\mu_{\omega}\right\}_{\omega \in \Omega}$ will be called a $\tau$ basic family if it has the following properties:

(i) For each $\omega \in \Omega, \mu_{\omega}$ is an FSM on $\mathscr{M}^{\tau(\omega)}$ and the map

$$
\omega \longrightarrow\left\{\begin{array}{cl}
\mu_{\omega}(A) & \text { if } \omega \in\{\tau \leqq t\} \text { and } A \in \mathscr{C}^{t}, \\
0 & \text { if } \omega \in\{\tau>\tau\},
\end{array}\right.
$$

from $\Omega$ into $R$, is $\mathscr{H}_{t}^{s}$-measurable for all $t \geqq s$ and all $A \in \mathscr{M}^{t}$.

(ii) For each $\omega \in \widetilde{\Omega}=\{\tau<\infty\}$ the complement of

$$
\Omega_{\omega}=\left\{\omega^{\prime} \in \Omega: X\left(\tau(\omega), \omega^{\prime}\right)=X(\tau(\omega), \omega)\right\}
$$

is a $\mu_{\omega}$-null set of $\mathscr{H}^{\tau(\omega)}$.

(iii) For $\omega \in \Omega-\widetilde{\Omega}$ and $A \in \mathscr{M}^{\infty}, \mu_{\omega}(A)=I_{A}(\omega)$.

For each $\omega \in \Omega$ the set function $\mu_{\omega}^{\prime}$ is defined on $\left(\Omega, \mathscr{C}^{s}\right)$ as follows: 


$$
\begin{aligned}
& \mu_{\omega}^{\prime}\left(\left\{X\left(t_{0}\right) \in \Gamma_{0}, \cdots, X\left(t_{n}\right) \in \Gamma_{n}\right\}\right) \\
& =\left\{\begin{array}{r}
I_{\left\{X\left(t_{i}\right) \in \Gamma_{i} ; i=0, \cdots, 1\right\}}(\omega) \mu_{\omega}\left(\left\{X\left(t_{i}\right) \in \Gamma_{i} ; i=\mathfrak{l}+1, \cdots, n\right\}\right) \\
\text { if } \omega \in\left\{t_{\mathfrak{r}} \leqq \tau<t_{\mathfrak{1}+1}\right\} \text { and } \mathfrak{l}=0,1, \cdots, n-1, \\
I_{\left\{X\left(t_{i}\right) \in \Gamma_{i} ; i=0, \cdots, n\right\}}(\omega) \mu_{\omega}(\Omega) \\
\text { if } \omega \in\left\{\tau \geqq t_{n}\right\},
\end{array}\right.
\end{aligned}
$$

with $0 \leqq s=t_{0}<t_{1}<\cdots<t_{n}, n \geqq 1$ and $\Gamma_{0}, \cdots, \Gamma_{n} \in \mathscr{S}$.

LEMma 5.3. For each $\omega \in \Omega, \mu_{\omega}^{\prime}$ is a countably additive set function on the semifield $\mathscr{C}^{3}$.

Proof. Fix $\omega_{0} \in \Omega$ and put $\tau_{0}=\tau\left(\omega_{0}\right)$. Let $C=\left\{X\left(t_{i}\right) \in \Gamma_{i} ; i=\right.$ $0, \cdots, n\}$ with $s=t_{0}<t_{1}<\cdots<t_{n}$ be a set in $\mathscr{C}^{s}$.

Suppose $\tau_{0} \in\left[t_{\mathrm{r}}, t_{\mathrm{t}+1}\right)$ where $0 \leqq \mathfrak{l} \leqq n-1$. We can write $C=A B$ with $A=\left\{X\left(t_{i}\right) \in \Gamma_{i} ; i=0, \cdots, \mathfrak{l}\right\}$ and $B=\left\{X\left(t_{i}\right) \in \Gamma_{i} ; i=\mathfrak{l}+1, \cdots, n\right\}$. Now $A \in \mathscr{C}_{\tau_{0}}^{s}$ and $B \in \mathscr{C}^{\tau_{0}+}$ where $\mathscr{C}^{\tau_{0}+}$ is the collection of sets of the form $\left\{X\left(u_{1}\right) \in \Delta_{1}, \cdots, X\left(u_{n}\right) \in \Delta_{n}\right\}$ with $\tau_{0}<u_{1}<\cdots<u_{n}, n \geqq 1$ and $\Delta_{1}, \cdots, \Delta_{n}$ Borel sets of $S$. Clearly, $\mu_{\omega_{0}}^{\prime}(C)=I_{A}\left(\omega_{0}\right) \mu_{\omega_{0}}(B)$.

If $\tau_{0} \in\left[t_{n}, \infty\right), C=A B$ with $A=C \in \mathscr{C}_{\tau_{0}}^{s}$ and $B=\Omega \in \mathscr{C}^{\tau_{0}+}$. If $\tau_{0}=\infty, C=A B$ with $A=C \in \mathscr{C}_{\tau_{0}}^{s}=\mathscr{C}_{\infty}^{s}=\mathscr{C}^{s}$ and $B=\Omega \in \mathscr{C}^{\tau_{0}+}=\mathscr{C}^{\infty}$.

In both cases $\mu_{\omega_{0}}^{\prime}(C)=I_{A}\left(\omega_{0}\right) \mu_{\omega_{0}}(B)$. To show countable additivity of $\mu_{\omega_{0}}^{\prime}$ on $\mathscr{C}^{s}$, let $C=\bigcup_{k=1}^{\infty} A_{k} B_{k}=A B \in \mathscr{C}^{s}$, where $A_{1} B_{1}, A_{2} B_{2}, \cdots$ are disjoint members of $\mathscr{C}^{s}$ with $A_{k} \in \mathscr{C}_{\tau_{0}}^{s}, B_{k} \in \mathscr{C}^{\tau_{0}+}, A \in \mathscr{C}_{\tau_{0}}^{s}$ and $B \in \mathscr{C}^{\tau_{0}+}$. Clearly, $\mu_{\omega_{0}}^{\prime}\left(\bigcup_{k} A_{k} B_{k}\right)=I_{A}\left(\omega_{0}\right) \mu_{\omega_{0}}(B)$.

In the case that $\omega_{0} \in \Omega-\widetilde{\Omega}$ countable additivity is obvious. Now let $\omega_{0} \in \widetilde{\Omega}$ and note that $\left|\mu_{\omega_{0}}\right|\left(\Omega_{0}^{c}\right)=0$, where $\Omega_{0}=\Omega_{\omega_{0}}$. If $\omega_{0} \in A \cap \widetilde{\Omega}$, $\mu_{\omega_{0}}^{\prime}\left(\mathrm{U}_{k} A_{k} B_{k}\right)=\mu_{\omega_{0}}(B)$ and $\sum_{k} \mu_{\omega_{0}}^{\prime}\left(A_{k} B_{k}\right)=\sum_{k} I_{A_{k}}\left(\omega_{0}\right) \mu_{\omega_{0}}\left(B_{k}\right)$. Let $J_{0}=$ $\left\{k: \omega_{0} \in A_{k}\right\}$. Thus if $J_{0} \neq \varnothing$, we have to show $\mu_{\omega_{0}}(B)=\sum_{k \in J_{0}} \mu_{\omega_{0}}\left(B_{k}\right)$ and if $J_{0}=\varnothing, \mu_{\omega_{0}}(B)=0$.

Let $J_{0} \neq \varnothing$. We claim $\Omega_{0} B_{k} \cap \Omega_{0} B_{k^{\prime}}=\varnothing$ if $k, k^{\prime} \in J_{0}$ and $k=k^{\prime}$. Suppose $\omega \in \Omega_{0} B_{k} \cap \Omega_{0} B_{k^{\prime}}$. There exists an $\omega_{1}$ such that

$$
\omega_{1}= \begin{cases}\omega_{0} & \text { on }\left[s, \tau_{0}\right] \\ \omega & \text { on }\left[\tau_{0}, \infty\right)\end{cases}
$$

Now $\omega_{0} \in A_{k} A_{k^{\prime}}$ and it follows that $\omega_{1} \in \Omega_{0} A_{k} A_{k^{\prime}} B_{k} B_{k^{\prime}}$, a contradiction. By a similar kind of argument it is seen that $\Omega_{0} B=\bigcup_{k \in J_{0}} \Omega_{0} B_{k}$. It follows that $\mu_{\omega_{0}}(B)=\sum_{k \in J_{0}} \mu_{\omega_{0}}\left(B_{k}\right)$. The remainder of the proof is along similar lines.

Lemma 5.4. For each $\omega \in \Omega$, $\mu_{\omega}^{\prime}$ has a unique extension to an FSM on $\mathscr{M}^{s}$. The total variation on $\Omega$ of this FSM does not exceed $\left|\mu_{\omega}\right|(\Omega)$.

Proof. Fix $\omega_{0} \in \Omega$ and put $\tau_{0}=\tau\left(\omega_{0}\right) . \quad \mu_{\omega_{0}}^{\prime}$ has a unique countably 
additive extension to the field $\mathscr{F}$ generated by $\mathscr{C}^{s}$. Denoting this by $\mu_{\omega_{0}}^{\prime}$, we have

$$
\left|\mu_{\omega_{0}, \sigma}^{\prime}\right|(\Omega)=\sup \sum_{k=1}^{n} \mid \mu_{\omega_{0}}^{\prime}\left(E_{k}\right),
$$

where the sup is taken over all finite sequences $\left\{E_{k}\right\}$ of disjoint sets in $\mathscr{C}^{s}$. We can write $E_{k}=A_{k} B_{k}$ with $A_{k} \in \mathscr{C}_{\tau_{0}}^{s}$ and $B_{k} \in \mathscr{C}^{\tau_{0}+}$ for $k=1, \cdots, n$. It follows that $\left|\mu_{\omega_{0}, \sigma}^{\prime}\right|(\Omega) \leqq\left|\mu_{\omega_{0}}\right|(\Omega)$. Thus $\mu_{\omega_{0}}^{\prime}$ has a unique extension to an FSM on $\mathscr{C}^{s}$.

REMARK 5.5. From now on $\mu_{\omega}^{\prime}$ will denote the extension to $\mathscr{C l}^{s}$ and $\left\{\mu_{\omega}^{\prime}\right\}_{\omega \in \Omega}$ will be called the family of FSM's associated with the $\tau$-basic family $\left\{\mu_{\omega}\right\}_{\omega \in \Omega}$. In Lemma 5.9 we will see that $\mu_{\omega, \mu \sim(\omega)}^{\prime}=\mu_{\omega}$ and it follows that $\left|\mu_{\omega}^{\prime}\right|(\Omega)=\left|\mu_{\omega}\right|(\Omega)$.

Lemma 5.6. The map $\omega \rightarrow \mu_{\omega}^{\prime}(B)$, from $\Omega$ into $R$, is . /ls-measurable for all $B \in \mathbb{l l}^{s}$.

Proof. It is easy to see that $\omega \rightarrow \mu_{\omega}^{\prime}(C)$ is . $\iota_{s}^{s}$-measurable for each $C \in \mathscr{C}^{s}$. By the monotone class theorem the proof is completed.

Lemma 5.7. Let $\omega \in \Omega$. Then for all $A \in \mathbb{l l}_{\mathrm{s}}^{\mathrm{s}}$ and $B \in \mathscr{C l}^{\mathrm{s}}$,

$$
\mu_{\omega}^{\prime}(A B)=I_{A}(\omega) \mu_{\omega}^{\prime}(B) \text {. }
$$

Proof. Fix $\omega_{0} \in \Omega$ and call $\tau\left(\omega_{0}\right)=\tau_{0}$. If $A=\{X(u) \in \Gamma\}$ with $s \leqq$ $u \leqq \tau_{0}\left(s \leqq u<\infty\right.$ if $\left.\tau_{0}=\infty\right)$ and $\Gamma \in \mathscr{S}$, then $\mu_{\omega_{0}}^{\prime}(A B)=I_{A}\left(\omega_{0}\right) \mu_{\omega_{0}}^{\prime}(B)$ for all $B \in \mathscr{C}^{s}$. By induction it follows that $\mu_{\omega_{0}}^{\prime}(A B)=I_{A}\left(\omega_{0}\right) \mu_{\omega_{0}}^{\prime}(B)$ for all $A \in \mathscr{C}_{:_{0}}^{s}$ and $B \in \mathscr{C}^{s}$. By a monotone class argument, $\mu_{\omega_{0}}^{\prime}(A B)=$ $I_{A}\left(\omega_{0}\right) \mu_{\omega_{0}}^{\prime}(B)$ for all $A \in \mathscr{C}_{\tau_{0}}^{s}$ and $B \in \mathscr{Z}^{s}$.

Now let $A \in \mathscr{C}_{\text {s }}^{s}$ and $B \in \mathscr{C}^{s}$. Since both $A \cap\left\{\tau=\tau_{0}\right\}$ and $\left\{\tau=\tau_{0}\right\}$ are in. $/ l_{\tau_{0}}^{s}$ it is easily seen that $\mu_{\omega_{0}}^{\prime}(A B)=I_{A}\left(\omega_{0}\right) \mu_{\omega_{0}}^{\prime}(B)$.

Lemma 5.8. For each $\omega \in \Omega, \mu_{\omega}^{\prime}(B)=\mu_{\omega}(B)$ for all $B \in \mathscr{l l}^{-(\omega)}$.

Proof. The assertion is obvious if $\omega \in \Omega-\widetilde{\Omega}$. Now let $\omega_{0} \in \widetilde{\Omega}$. If $B=\left\{X\left(t_{i}\right) \in \Gamma_{i} ; i=1, \cdots, m\right\}$ with $\tau_{0}=\tau\left(\omega_{0}\right)<t_{1}<\cdots<t_{m}$ and $\Gamma_{1}, \cdots, \Gamma_{m} \in \mathscr{S}$, then $\mu_{\omega_{0}}^{\prime}(B)=\mu_{\omega_{0}}(B)$ and thus the assertion is true for all $B \in \sigma\left(\mathscr{C}^{0^{0+}}\right)$. But $\sigma\left(\mathscr{C}^{i_{0}+}\right)=\mathscr{C}^{i_{0}}$ and the lemma is proved.

6. The patching theorem. In this section $S$ will be a separable metric space. The $\sigma$-field $\mathscr{C}^{\mathrm{s}}$ then is countably generated.

LeMma 6.1. Let $\left\{\mu_{\omega}\right\}_{\omega \in \Omega}$ be a $\tau$-basic family of FSM's and $\left\{\mu_{\omega}^{\prime}\right\}_{\omega \in \Omega}$ its associated family. For each $B \in \mathscr{H}^{s}$, the map $\omega \rightarrow\left|\mu_{\omega}^{\prime}\right|(B)$, from 
$\Omega$ into $R$, is $/ l_{\tau}^{s}$-measurable.

Proof. See proof of Corollary 3.2.

THEOREM 6.2 (Patching theorem). Let $\mu$ be an FSM on $\left(\Omega, \mathscr{C l}_{\tau}^{s}\right)$ and $\left\{\mu_{\omega}\right\}_{\omega \in \Omega}$ a $\tau$-basic family of FSM's. Assume, moreover, that for all $\omega \in \Omega, \mu_{\omega} \neq 0$ and that the map $\omega \rightarrow\left|\mu_{\omega}\right|(\Omega) / \mu_{\omega}(\Omega)$, from $\Omega$ into the extended real line, is $\mu$-integrable. If $\left\{\mu_{\omega}^{\prime}\right\}_{\omega \in \Omega}$ is the family of FSM's associated with $\left\{\mu_{\omega}\right\}_{\omega \in \Omega}$, then the intgral

$$
\mu^{\prime}(B)=\int \frac{\mu_{\omega}^{\prime}(B)}{\mu_{\omega}^{\prime}(\Omega)} d \mu(\omega),
$$

with $B \in \mathscr{C}^{s}$, defines an FSM $\mu^{\prime}$ on $\mathscr{M}^{s}$. $\mu^{\prime}$ has the properties:

(i ) $\mu^{\prime}(A)=\mu(A)$ for all $A \in \mathscr{l}_{\tau}^{s}$ i.e., $\mu=\mu_{:}^{\prime}$, the restriction of $\mu^{\prime}$ to $\mathscr{L}_{\tau}^{s}$.

$$
\mu^{\prime}(A \cap B)=\int_{A} \frac{\mu_{\omega}^{\prime}(B)}{\mu_{\omega}^{\prime}(\Omega)} d \mu_{t}^{\prime}(\omega)
$$

for all $A \in \mathscr{L}_{\tau}^{s}$ and $B \in \mathscr{M}^{s}$.

Proof. If $\left\{B_{k}\right\}_{1}^{\infty}$ is any sequence of disjoint sets in $\mathscr{C}^{s}$, then

$$
\sum_{1}^{\infty} \int\left|\frac{\mu_{\omega}^{\prime}\left(B_{k}\right)}{\mu_{\omega}^{\prime}(\Omega)}\right| d|\mu|(\omega) \leqq \int \frac{\left|\mu_{\omega}\right|(\Omega)}{\left|\mu_{\omega}(\Omega)\right|} d|\mu|(\omega) .
$$

By the dominated convergence theorem $\mu^{\prime}$ is countably additive and thus an FSM on $\mathscr{C}^{s}$. By Lemma $5.7 \mu^{\prime}(A)=\mu(A)$ for $A \in \mathscr{M}_{\tau}^{s}$ and also (ii) follows.

THEOREM 6.3. Let $\mu$ and $\mu^{\prime}$ be as in Theorem 6.2. There exists an RCD, $Q^{\prime}$, of $\mu^{\prime}$ given $\mathscr{H}_{\tau}^{s} . Q^{\prime}$ is unique in the sense of Lemma 1.5. Furthermore, if $N=\left\{\omega \in \Omega: \mu_{\omega}(\Omega)=0\right\}$, then for all $\omega \in \Omega-N$ :

(i) $Q^{\prime}(\omega, B)=\mu_{\omega}(B) / \mu_{\omega}(\Omega)$ for all $B \in \mathscr{C}^{\tau(\omega)}$.

(ii) $Q^{\prime}\left(\omega, A_{\omega}\right)=1$ and $\left|Q^{\prime}\right|\left(\omega, A_{\omega}^{c}\right)=0$, where $A_{\omega}$ is the atom of $\mathscr{L}_{\tau}^{\mathrm{s}}$ containing $\omega$.

Proof. Define a function $Q^{\prime}: \Omega \times \mathscr{C}^{3} \rightarrow R$ by

$$
Q^{\prime}(\omega, B)= \begin{cases}\frac{\mu_{\omega}^{\prime}(B)}{\mu_{\omega}^{\prime}(\Omega)} & \text { if } \omega \notin N \text { and } B \in \mathscr{M}^{s}, \\ P(B) & \text { if } \omega \in N \text { and } B \in \mathscr{M}^{s},\end{cases}
$$

where $P$ is a fixed but arbitrary probability measure on $\mathscr{M}^{s}$. Clearly, $Q^{\prime}$ is an RCD of $\mu^{\prime}$ given $\mathscr{l}_{\tau}^{s}$. As $\mathscr{L}^{s}$ is countably generated the second assertion follows. By Lemma 5.8, $Q^{\prime}(\omega, B)=$ 
$\mu_{\omega}(B) / \mu_{\omega}(\Omega)$ for all $B \in \mathscr{C}^{\tau(\omega)}$ if $\omega \notin N$. The last part is an immediate consequence of Lemma 5.7.

THEOREM 6.4. The FSM $\mu^{\prime}$ defined in Theorem 6.2 in terms of $\left\{\mu_{\omega}\right\}_{\omega \in \Omega}$ and $\mu$ is the only FSM on $\mathscr{A l}^{s}$ such that

(i ) $\mu^{\prime}(A)=\mu(A)$ for all $A \in$. $\ell_{\tau}^{s}$,

(ii) $\mu^{\prime}$ has an RCD, $Q^{\prime}$, given. $\mathscr{L}_{\tau}^{s}$,

(iii) $Q^{\prime}(\omega, B)=\mu_{\omega}(B) / \mu_{\omega}(\Omega)$ for all $B \in \mathscr{l}^{-(\omega)}$ except for $\omega$ in a $\mu^{\prime}-$ null set of .llis. $_{s}^{s}$.

Proof. Suppose $\bar{\mu}$ is another FSM on $\mathscr{L}^{s}$ satisfying (i), (ii) and (iii), and let $\bar{Q}$ be its RCD given $\mathscr{C l}_{:}^{s}$. Let $B=\left\{X\left(t_{i}\right) \in \Gamma_{i} ; i=0, \cdots, n\right\}$ with $s=t_{0}<t_{1}<\cdots<t_{n}$ and $\Gamma_{0}, \cdots, \Gamma_{n} \in \mathscr{S}$. Put $A_{1}=\left\{t_{1} \leqq \tau<t_{\imath+1}\right\}$ for $\mathfrak{l}=0,1, \cdots, n-1$ and $A_{n}=\left\{\tau \geqq t_{n}\right\}$. Clearly, $A_{\mathfrak{l}} \in \mathscr{A}_{\tau}^{s}$ for $\mathfrak{l}=$ $0,1, \cdots, n$. Now it follows from Lemmas 5.7 and 5.8 , and (i) and (iii) that

$$
\begin{aligned}
\mu^{\prime}(B) & =\sum_{\imath=0}^{n} \int_{A_{\mathfrak{l}}} \frac{\mu_{\omega}^{\prime}(B)}{\mu_{\omega}^{\prime}(\Omega)} d \mu_{\tau}^{\prime}(\omega) \\
& =\sum_{\imath=0}^{n} \bar{\mu}\left(A_{\imath} \cap B\right)=\bar{\mu}(B) .
\end{aligned}
$$

Thus $\mu^{\prime}$ and $\bar{\mu}$ agree on $\mathscr{C}^{s}$ and hence on $\mathscr{l l}^{s}$.

7. The Jordan decomposition of $\mu_{\omega^{*}}^{\prime}$ Let $\left\{\mu_{\omega}\right\}_{\omega \in \Omega}$ be a $\tau$-basic family of FSM's and let $\mu_{\omega}=\mu_{\omega}^{+}-\mu_{\omega}^{-}$be the Jordan decomposition of $\mu_{\omega}$. For each $\omega \in \widetilde{\Omega}, \mu_{\omega}^{+}\left(\Omega_{\omega}^{c}\right)=\mu_{\omega}^{-}\left(\Omega_{\omega}^{c}\right)=0$ and we define $\mu_{\omega}^{\prime}, \mu_{\omega}^{+\prime}$ and $\mu_{\omega}^{-1}$ on $\left(\Omega, \mathscr{C}^{s}\right)$ as in 5.2 and extend to $\left(\Omega, \mathscr{C}^{s}\right)$. For $\omega \in \Omega-\widetilde{\Omega}$ put $\mu_{\omega}^{\prime}(A)=\mu_{\omega}^{+\prime}(A)=I_{A}(\omega)$ and $\mu_{\omega}^{-1}(A)=0$ for all $A \in \mathbb{C l}^{s}$. We have the following.

Lemma 7.1. If $\mu_{\omega}^{\prime}=\mu_{\omega}^{\prime+}-\mu_{\omega}^{\prime-}$ is the Jordan decomposition of $\mu_{\omega}^{\prime}$, then for all $\omega \in \Omega$ and $A \in \mathscr{L l}^{s}$

$$
\mu_{\omega}^{\prime+}(A)=\mu_{\omega}^{+\prime}(A) \text { and } \quad \mu_{\omega}^{\prime-}(A)=\mu_{\omega}^{-\prime}(A) .
$$

Proof. There exists for each $\omega \in \Omega$ a set $D_{\omega} \in \mathscr{C l}^{-(\omega)}$ such that $\mu_{\omega}^{+}(A)=\mu_{\omega}\left(A \cap D_{\omega}\right)$ and $\mu_{\omega}^{-}(A)=-\mu_{\omega}\left(A \cap D_{\omega}^{c}\right)$ for all $A \in \mathscr{C}^{\tau(\omega)}$. Let $A \in \mathscr{C}^{s}$. It is not hard to see that $\mu_{\omega}^{\prime}\left(A \cap D_{\omega}\right)=\mu_{\omega}^{+\prime}(A)$ and $\mu_{\omega}^{\prime}\left(A \cap D_{\omega}^{c}\right)=-\mu_{\omega}^{-\prime}(A)$. It follows that $\mu_{\omega}^{\prime}\left(A \cap D_{\omega}\right)=\mu_{\omega}^{+\prime}(A)$ and $\mu_{\omega}^{\prime}\left(A \cap D_{\omega}^{c}\right)=-\mu_{\omega}^{-\prime}(A)$ for all $A \in \mathscr{L}^{s}$. Hence $\mu_{\omega}^{\prime+}=\mu_{\omega}^{+\prime}$ and $\mu_{\omega}^{\prime-}=\mu_{\omega}^{\prime \prime}$.

Lemma 7.2. Let $\left\{\mu_{\omega}^{\prime}\right\}_{\omega \in \Omega}$ be the family of FSM's on $\left(\Omega, \mathscr{C l}^{s}\right)$ associated with $\left\{\mu_{\omega}\right\}_{\omega \in \Omega}$. If $S$ is a separable metric space, then the maps $\omega \rightarrow \mu_{\omega}^{\prime+}(B)$ and $\omega \rightarrow \mu_{\omega}^{\prime-}(B)$, from $\Omega$ into $R$, are $\mathscr{L}_{\tau}^{s}$-measura- 
ble for each $B \in \mathscr{A l}^{s}$.

Proof. For all $B \in \mathscr{C l}^{s}$ and $\omega \in \Omega$ we can write

$$
\begin{aligned}
& \mu_{\omega}^{\prime+}(B)=\frac{1}{2}\left\{\left|\mu_{\omega}^{\prime}\right|(B)+\mu_{\omega}^{\prime}(B)\right\}, \\
& \mu_{\omega}^{\prime}(B)=\frac{1}{2}\left\{\left|\mu_{\omega}^{\prime}\right|(B)-\mu_{\omega}^{\prime}(B)\right\} .
\end{aligned}
$$

Now apply Lemmas 5.6 and 6.1 .

REMARK 7.3. It is now obvious that under the conditions of Theorem 6.2 we can write for all $A \in \mathscr{L}_{\tau}^{s}$ and $B \in \mathscr{M}^{s}$ :

$$
\int_{A} \frac{\mu_{\omega}^{\prime}(B)}{\mu_{\omega}^{\prime}(\Omega)} d \mu(\omega)=\int_{A} \frac{\mu_{\omega}^{+\prime}(B)}{\mu_{\omega}^{\prime}(\Omega)} d \mu(\omega)-\int_{A} \frac{\mu_{\omega}^{-\prime}(B)}{\mu_{\omega}^{\prime}(\Omega)} d \mu(\omega) .
$$

8. The reverse of patching. From now on $S$ will be a complete separable metric space. Let $0 \leqq a \leqq b<\infty$ and let $C_{b}^{a}=$ $C([a, b] ; S)$ be the space of continuous functions on $[a, b]$ with values in $S$. It is well known that $C_{b}^{a}$ with the uniform metric is a complete separable metric space and that the Borel $\sigma$-field on $C_{b}^{a}$ coincides with the $\sigma$-field generated by the coordinate projections, e.g., see [9]. Let $D_{b}^{r}=D([a, b] ; S)$ be the space of functions on $[a, b]$ that are right-continuous and have left-hand limits, with values in $S$. If $S=R$, it is shown in [1] that $D_{b}^{x}$ with the Skorohod topology is a separable completely metrizable space. Its Borel $\sigma$-field coincides with the $\sigma$-field generated by the coordinate projections. If $S$ is any complete separable metric space the same can be shown, see for instance [8]. That $\left(\Omega, \mathscr{C}^{s}\right)$ is standard is then an easy application of Theorem 2.3.

If $\tau$ is an $s$-Markov time as in 5.1, it is shown in [6] that $\left(\Omega, \mathscr{C}_{\tau}^{s}\right)$ is standard in the case that $\Omega=C\left([0, \infty] ; R^{d}\right.$ ) (see also [7]). Along similar lines it can be shown that $\left(\Omega, \mathscr{C}_{r}^{s}\right)$ is standard if $\Omega=C([0, \infty) ; S)$ or $\Omega=D([0, \infty) ; S)$ with $S$ any complete separable metric space. In fact $\mathscr{C}_{\tau}^{s}$ is generated by the collection of sets of the form $\left\{X\left(t_{1} \wedge \tau\right) \in \Gamma_{1}, \cdots, X\left(t_{n} \wedge \tau\right) \in \Gamma_{n}\right\}$ with $t_{1}, \cdots, t_{n}$ points in $[s, \infty), \quad \Gamma_{1}, \cdots, \Gamma_{n} \in \mathscr{S}$ and $n \geqq 1$. Also $\Omega(\tau)=\{\omega: X(t, \omega)=$ $X(t \wedge \tau(\omega), \omega)$ for all $t \geqq 0\}$ is a set in $\mathscr{C}^{s}$ (compare p. 395 of [6]). If $\Psi_{\tau}: \Omega \rightarrow \Omega$ is defined by $\Psi_{\tau}(\omega)(t)=\omega(t \wedge \tau(\omega))$ for all $\omega \in \Omega$ and $t \geqq 0$, then $\Psi_{\tau}^{-1}\left(\mathscr{C}^{s} \cap \Omega(\tau)\right)=\mathscr{C}_{\tau}^{s}$. For details see [8].

We have the following extension of Theorem 1.3.4 on p. 34 of [7].

THEOREM 8.1. Let $\mu$ be an FSM on $\left(\Omega, \mathscr{C}^{s}\right)$. The condition 
that a $\mu_{\mathscr{N}_{\tau}^{s}-n u l l}$ set of $\Omega$ is a $\mu_{\text {-null set, }}$ is necessary and sufficient for the existence of an RCD of $\mu$ given $\mathscr{A}_{t}^{s}$. If the condition is satisfied there exists an RCD, $Q$, of $\mu$ given $\mathscr{C}_{:}^{s}$ such that

$$
Q(\omega, A B)=I_{A}(\omega) Q(\omega, B)
$$

for all $\omega \in \Omega, A \in \mathscr{H}_{\tau}^{s}$ and $B \in \mathscr{M}^{s}$.

Proof. The first assertion follows from Theorem 3.1. Assume the condition is satisfied and let $Q^{\prime}$ be an RCD of $\mu$ given $\mathscr{L}_{\tau}^{s}$. By Corollary 3.3, there exists a $\mu$-null set $N \in \mathscr{C}_{\tau}^{s}$ such that for all $A \in \mathscr{C l}_{\tau}^{s}$ and $B \in \mathscr{C}^{s}, Q^{\prime}(\omega, A B)=I_{A}(\omega) Q^{\prime}(\omega, B)$ if $\omega \notin N$. Define $Q$ on $\Omega \times \mathscr{C}^{s}$ by

$$
Q(\omega, B)= \begin{cases}Q^{\prime}(\omega, B) & \text { for } B \in \mathscr{C}^{s} \text { and } \omega \notin N, \\ I_{B}\left(\Psi_{\tau}(\omega)\right) & \text { for } B \in \mathscr{C}^{s} \text { and } \omega \in N .\end{cases}
$$

Clearly, $Q$ has the required properties.

TheOREm 8.2. Let $\mu^{\prime}$ be an FSM on $\left(\Omega, \mathscr{l}^{s}\right)$ and let $\mu$ be its restriction to $\mathscr{L}_{s}^{s}$. Assume that a $\mu$-null set of $\Omega$ is also a $\mu^{\prime}$ null set. Then there exists a $\tau$-basic family of FSM's $\left\{\mu_{\omega}\right\}_{\omega \in \Omega}$ with the properties (i) $\mu_{\omega}(\Omega)=1$ for all $\omega \in \Omega$, (ii) the map $\omega \rightarrow\left|\mu_{\omega}\right|(\Omega)$ is $\mu$-integrable and (iii) $\mu^{\prime}$ coincides with the FSM obtained by patching $\mu$ and $\left\{\mu_{\omega}\right\}_{\omega \in \Omega}$ together as in Theorem 6.2.

Proof. Let $Q^{\prime}$ be an RCD of $\mu^{\prime}$ given $\mathscr{A}_{\tau}^{s}$ such that $Q^{\prime}(\omega, A B)=$ $I_{A}(\omega) Q^{\prime}(\omega, B)$ for all $\omega \in \Omega, A \in \mathscr{L}_{\tau}^{s}$ and $B \in \mathscr{L}^{s}$. Define the family of FSM's $\left\{\mu_{\omega}\right\}_{\omega \in \Omega}$ as follows: For each $\omega \in \Omega, \mu_{\omega}(A)=Q^{\prime}(\omega, A)$ for all $A \in \mathscr{C}^{\tau(\omega)} . \quad\left\{\mu_{\omega}\right\}_{\omega \in \Omega}$ is a $\tau$-basic family. Let $\left\{\mu_{\omega}^{\prime}\right\}_{\omega \in \Omega}$ be the family of associated FSM's on $\left(\Omega, \mathscr{C}^{s}\right)$. It is easily seen that $\mu_{\omega}^{\prime}(B)=$ $Q^{\prime}(\omega, B)$ for all $\omega \in \Omega$ and $B \in \mathscr{C}^{s}$. It follows that $\left\{\mu_{\omega}\right\}_{\omega \in \Omega}$ has properties (ii) and (iii).

9. Patching countably many times. Let $\tau_{0} \leqq \tau_{1} \leqq \tau_{2} \leqq \cdots$ be a sequence of $s$-Markov times, from $\Omega$ into $[s, \infty]$, with respect to the family of $\sigma$-fields $\left\{\mathscr{L}_{t}^{s}, t \geqq s\right\}$. For each $n=0,1,2, \cdots$ let $\left\{\mu_{n \omega}\right\}_{\omega \in \Omega}$ be a $\tau_{n}$-basic family of FSM's and $\left\{\mu_{n_{\omega}}^{\prime}\right\}_{\omega \in \Omega}$ its associated family of FSM's on $\left(\Omega, \mathscr{C}^{s}\right)$. Assume, moreover, that

$$
\alpha=\prod_{0}^{\infty} \sup _{\omega \in\lrcorner} \frac{\left|\mu_{n \omega}\right|(\Omega)}{\left|\mu_{n \omega}(\Omega)\right|}<\infty .
$$

For $n=0,1,2, \cdots$ define the transition functions $f_{n+1}$, from $\left(\Omega, \mathscr{C}_{\tau_{n}}^{s}\right)$ to $\left(\Omega, \mathscr{C}_{s_{n+1}}^{s}\right)$, by $f_{n+1}(\omega, B)=\mu_{n_{\omega}}^{\prime}(B) / \mu_{n_{\omega}}^{\prime}(\Omega)$. Let $M_{n}=$ $M\left(\Omega, \mathscr{L}_{\tau_{n}}^{s}\right)$ be the Banach space of all FSM's on $\left(\Omega, \mathscr{C}_{\tau_{n}}^{s}\right)$. If $\mathscr{A}^{*}=$ 
$\sigma\left(\bigcup_{0}^{\infty} \mathscr{A}_{\tau_{n}}^{s}\right)$, let $M^{*}$ be the Banach space of FSM's on $\left(\Omega, \mathscr{M}^{*}\right)$. With $f_{m, n}$ defined as in 4.2 and $L_{m, n}$ as in 4.3 we can apply Theorem 4.4.

ACKNowledgments. It is a pleasure to thank R. B. Kirk and P. Feinsilver for helpful conversations.

\section{REFERENCES}

1. P. Billingsley, Convergence of Probability Measures, John Wiley, New York, 1968.

2. N. Dunford and J. T. Schwartz, Linear Operators, John Wiley (Interscience), New York, Part I.

3. K. J. Hochberg, A signed measure on path space related to Wiener measure, Ann. Probability, 6 (1978), 433-458.

4. V. Yu. Krylov, Some properties of the distribution corresponding to the equation $\partial u / \partial t=(-1)^{q+1} \partial^{2 q} u / \partial x^{2 q}$, Soviet Math. Dokl., 1 (960), 760-763.

5. K. R. Parthasarathy, Probability Measures on Metric Spaces, Academic Press, New York, 1967.

6. D. W. Strook and S. R. S. Varadhan, Diffusion processes with continuous coefficients, I, Comm. Pure Appl. Math., XXII (1969), 345-400.

7. —, Multidimensional Diffusion Processes, Springer Verlag, Berlin-HeidelbergNew York, 1979.

8. A. J. Van Haagen, Finite signed measures on function spaces, dissertation for the $\mathrm{Ph}$. D degree, Southern Illinois University at Carbondale, (1979).

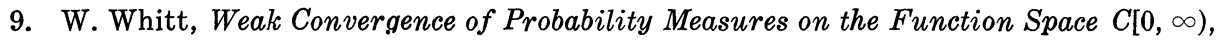
Ann. Math. Stat., 41 (1970), 939-944.

Received April 23, 1980.

19 ZeEburgerpad

1018 Ah Amsterdam, The Netherlands

Current address: Southern Illinois University

Carbondale, IL 62901 


\section{PACIFIC JOURNAL OF MATHEMATICS}

\section{EDITORS}

DONALD BABBITT (Managing Editor)

University of California

Los Angeles, CA 90024

HUGo RossI

University of Utah

Salt Lake City, UT 84112

C. C. MOORE and ANDREW OGG

University of California

Berkeley, CA 94720
J. DugundjI

Department of Mathematics

University of Southern California

Los Angeles, CA 90007

R. FinN and J. Milgram

Stanford University

Stanford, CA 94305

\section{ASSOCIATE EDITORS}
R. ARENS
E. F. BECKENBACH
B. H. NeumanN
F. WOLF
K. YOSHIDA

\section{SUPPORTING INSTITUTIONS}

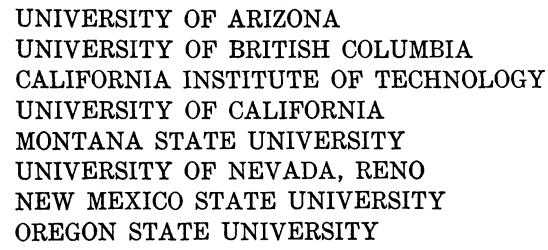

UNIVERSITY OF ARIZONA

UNIVERSITY OF BRITISH COLUMBIA

CALIFORNIA INSTITUTE OF TECHNOLOGY

UNIVERSITY OF CALIFORNIA

MONTANA STATE UNIVERSITY

UNIVERSITY OF NEVADA, RENO

NEW MEXICO STATE UNIVERSITY

OREGON STATE UNIVERSITY

\author{
UNIVERSITY OF OREGON \\ UNIVERSITY OF SOUTHERN CALIFORNIA \\ STANFORD UNIVERSITY \\ UNIVERSITY OF HAWAII \\ UNIVERSITY OF TOKYO \\ UNIVERSITY OF UTAH \\ WASHINGTON STATE UNIVERSITY \\ UNIVERSITY OF WASHINGTON
}

The Supporting Institutions listed above contribute to the cost of publication of this Journal, but they are not owners or publishers and have no responsibility for its content or policies.

Mathematical papers intended for publication in the Pacific Journal of Mathematics should be in typed form or offset-reproduced, (not dittoed), double spaced with large margins. Please do not use built up fractions in the text of the manuscript. However, you may use them in the displayed equations. Underline Greek letters in red, German in green, and script in blue. The first paragraph or two must be capable of being used separately as a synopsis of the entire paper. Please propose a heading for the odd numbered pages of less than 35 characters. Manuscripts, in triplicate, may be sent to any one of the editors. Please classify according to the scheme of Math. Reviews, Index to Vol. 39. Supply name and address of author to whom proofs should be sent. All other communications should be addressed to the managing editor, or Elaine Barth, University of California, Los Angeles, California, 90024.

50 reprints to each author are provided free for each article, only if page charges have been substantially paid. Additional copies may be obtained at cost in multiples of 50 .

The Pacific Journal of Mathematics is issued monthly as of January 1966. Regular subscription rate: $\$ 102.00$ a year (6 Vols., 12 issues). Special rate: $\$ 51.00$ a year to individual members of supporting institutions.

Subscriptions, orders for numbers issued in the last three calendar years, and changes of address shoud be sent to Pacific Journal of Mathematics, P.O. Box 969, Carmel Valley, CA 93924, U.S.A. Old back numbers obtainable from Kraus Per!odicals Co., Route 100, Millwood, NY 10546.

PUBLISHED BY PACIFIC JOURNAL OF MATHEMATICS, A NON-PROFIT CORPORATION

Printed at Kokusai Bunken Insatsusha (International Academic Printing Co., Ltd.). 8-8, 3-chome, Takadanobaba, Shinjuku-ku, Tokyo 160, Japan. 


\section{Pacific Journal of Mathematics}

\section{Vol. 95, No. $2 \quad$ October, 1981}

George E. Andrews, The Rogers-Ramanujan reciprocal and Minc's

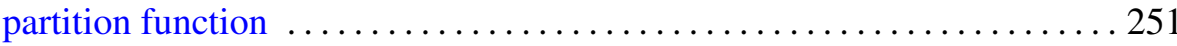

Allan Calder, William H. Julian, Ray Mines, III and Fred Richman,

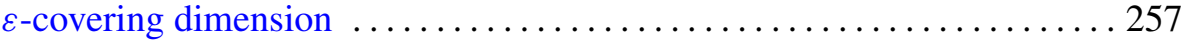

Thomas Curtis Craven and George Leslie Csordas, An inequality for the distribution of zeros of polynomials and entire functions $\ldots \ldots \ldots \ldots 263$

Thomas Jones Enright and R. Parthasarathy, The transfer of invariant

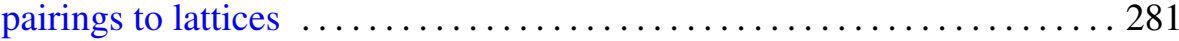

Allen Roy Freedman and John Joseph Sember, Densities and

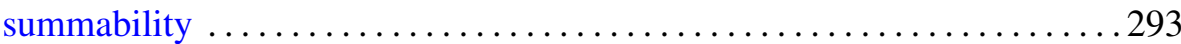

Robert Heller and Francis Aubra Roach, A generalization of a classical necessary condition for convergence of continued fractions . . . . . . 307

Peter Wilcox Jones, Ratios of interpolating Blaschke products ........... 311

V. J. Joseph, Smooth actions of the circle group on exotic spheres ........ 323

Mohd Saeed Khan, Common fixed point theorems for multivalued

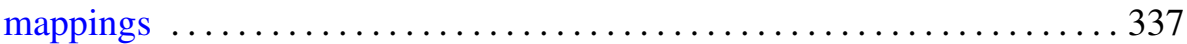

Samuel James Lomonaco, Jr., The homotopy groups of knots. I. How to

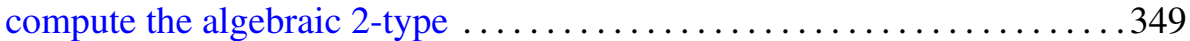

Louis Magnin, Some remarks about $C^{\infty}$ vectors in representations of connected locally compact groups ............................ 391

Mark Mandelker, Located sets on the line . . . . . . . . . . . . . . . . . 401

Murray Angus Marshall and Joseph Lewis Yucas, Linked quaternionic mappings and their associated Witt rings $\ldots \ldots \ldots \ldots \ldots \ldots \ldots \ldots . \ldots \ldots 11$

William Lindall Paschke, $K$-theory for commutants in the Calkin algebra

W. J. Phillips, On the relation $P Q-Q P=-i I$ 435

Ellen Elizabeth Reed, A class of Wallman-type extension. 443

Sungwoo Suh, The space of real parts of algebras of Fourier transforms 461 Antonius Johannes Van Haagen, Finite signed measures on function

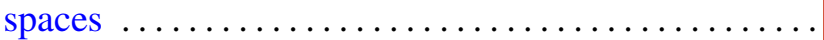

Richard Hawks Warren, Identification spaces and unique uniformity 